\title{
Surgery for spinal metastatic tumors: Prognostication systems in clinical practice (Review)
}

\author{
KALLIOPI ALPANTAKI ${ }^{1}$, ARGYRIOS IOANNIDIS ${ }^{2}$, KONSTANTINOS RAPTIS $^{3}$, \\ ELEFTHERIOS SPARTALIS ${ }^{4}$ and CHRISTOS KOUTSERIMPAS ${ }^{3}$
}

\author{
${ }^{1}$ Department of Orthopaedics, 'Venizeleion' General Hospital, Heraklion, Crete 71409; ${ }^{2}$ Department of General, \\ Laparoscopic, Oncologic and Robotic Surgery, Athens Medical Center, Athens 15125; ${ }^{3}$ Department of Orthopaedics and \\ Traumatology, '251' Hellenic Air Force General Hospital, Athens 11525; ${ }^{4}$ Laboratory of Experimental Surgery and \\ Surgical Research, University of Athens Medical School, Athens 11527, Greece
}

Received September 16, 2019; Accepted February 3, 2020

DOI: $10.3892 / \mathrm{mco} .2020 .2008$

\begin{abstract}
The management of spinal metastatic tumors is a matter of increasing clinical importance, as $20-40 \%$ of cancer patients have evidence of vertebral metastatic disease at the time of their passing and up to $20 \%$ develop neurological symptoms due to epidural spinal cord compression. The treatment of patients with spinal metastases is challenging, albeit palliative, and it requires a multidisciplinary approach. Accurate prediction of life expectancy of patients with cancer is of paramount importance for therapeutic strategy. Prognostication scoring systems were developed to aid clinicians to follow a more objective, safe and evidence-based approach with therapy selection and surgical intervention indications. In this context, the aim of the present review was to briefly discuss the evolution of scoring systems since their introduction in the early $90 \mathrm{~s}$ until today, their advantages and shortcomings, and the future requirements for personalized scoring in the era of modern oncology.
\end{abstract}

\section{Contents}

1. Introduction

2. Prognostication systems

3. New treatment options

4. NOMS algorithm

5. Future perspectives

6. Conclusions

Correspondence to: Dr Christos Koutserimpas, Department of Orthopaedics and Traumatology, '251' Hellenic Air Force General Hospital, Building 3b Kanellopoulou Avenue, Athens 11525, Greece E-mail: chrisku91@hotmail.com

Key words: spinal metastases, spine metastases surgery, prognostication system, NOMS, Tokuhashi scoring system

\section{Introduction}

The management of spinal metastatic tumors is a matter of increasing clinical importance, as $20-40 \%$ of cancer patients have evidence of vertebral metastatic disease at the time of their passing and up to $20 \%$ develop neurological symptoms due to epidural spinal cord compression. The extent of the problem is estimated to further increase, since the overall survival of nearly all malignant tumors has notably improved due to advances in chemotherapy, radiotherapy and targeted therapies and, recently, immunotherapy. The early detection of the disease, due to the availability of modern diagnostic imaging techniques, such as magnetic resonance (MR) and fluorodeoxyglucose positron emission tomography, also plays a significant role (1). The treatment of patients with spinal metastases is challenging, albeit palliative, and requires a multidisciplinary approach. Treatment, which ideally should be individually tailored, aims to preserve or restore neurological function and spinal stability and to improve the patients' pain and quality of life in a setting of a long-term local tumor control $(1,2)$.

Patients with metastatic spinal lesions are frequently referred to a spinal surgeon. The main reasons for referral include pain, neurological impairment and progressive deformity, all directly affecting the patient's quality of life, resulting in a markedly increased healthcare burden, particularly in case of paralysis. However, the referral pattern is often inconsistent, subject to local facilities (availability of specialists and treatment modalities), while the indications for surgery may be unjustified or even intuitive (3). Currently, the indications for surgical management of spinal metastases are summarized as follows: Neural compression secondary to retropulsed bone or spinal deformity, radioresistant tumors (e.g., renal cancer), radiation failure (progression of deficit during treatment or dose reaching spinal cord tolerance), spinal instability and intractable pain unresponsive to non-operative management (4).

Surgery for metastatic spinal disease is high-risk, with often unpredictable and adverse outcomes. Careful patient selection is necessary to ensure optimal outcome $(2,3)$. Aggressive decompression of the spinal canal through the 
posterior or ventral approaches, followed by stabilization using metal implants, has achieved better outcomes in terms of pain control and neurological restoration compared with simple laminectomy, with or without postoperative adjacent irradiation $(3,5)$. However, the success of extensive radical interventions is restricted by increased perioperative mortality and complication rates $(3,6)$. Therefore, prognostication instruments were introduced to predict survival and clearly determine the indications for surgical intervention (3).

In this context, we herein briefly review the literature on scoring systems for vertebral metastases and discuss their impact in current clinical practice. The present study is a descriptive, non-systematic review on the potential value of vertebral metastases scoring systems in clinical practice. A meticulous search through the PubMed and Cochrane Library databases was performed, and English, peer-reviewed articles were evaluated.

\section{Prognostication systems}

When managing patients with metastatic spinal disease, surgeons often exaggerate the effectiveness of surgical intervention and misjudge the patient's life expectancy, leading to inappropriate treatment. Accurate assessment of prognosis, prior to intervention, is of utmost importance for surgical treatment selection (7). Frequently, surgery is recommended for patients at high risk of intraoperative mortality, for those with a low likelihood of restoring neurological function, or for those whose life expectancy may be shorter than the anticipated recovery time (7-9). Therefore, selecting the type of treatment is complex, difficult and frequently debatable. The complexity of treatment also results from the multitude of available options, the heterogeneity of the patient population with respect to the biology of the primary tumor, the location and symptoms caused by the spinal lesions, as well as the patient's wishes, aspirations and functional status (7). Prognostication systems have been developed to assist oncologists and spinal surgeons in evaluating treatment options, their benefits (likelihood of improving neurological function, decreasing pain and restoring spine stability) and their disadvantages (surgical morbidity, mortality and recovery time) $(7,10)$. Ideally, a prognostication system would compare different management options, evaluate the outcome of surgical intervention based on survival, toxicity and cost-effectiveness, and propose a more rational, objective, safe and reproducible management $(3,7,10)$.

The Tokuhashi scoring system was introduced in 1989 as a 'scoring system for the preoperative evaluation of a patient's prognosis with a metastatic spinal tumor' (11) and it was revised in 2005 (7). Tomita et al, introduced an alternative scoring system in 2001 (12). Both Tokuhashi and Tomita scores take into consideration the type of primary tumor, the burden of bone secondary lesions and the presence of visceral metastases as critical prognostic factors. The Tokuhashi system acknowledges the significance of functional parameters, such as the ability to ambulate, while the Tomita score completely overlooks paralysis as factor for poor prognosis (7). Over the following years, a plethora of prognostication scoring systems were introduced, including the Bauer, modified Bauer, Van der Linden, Rades and Katagiri scores (7). Over several years, the reported scores practically attempted to evaluate the surgical indications and the radicality of the operation, taking survival as a prerequisite $(2,3)$. Their predictive value and clinical relevance have been assessed by several studies, but the results have been inconsistent $(3,8,9)$. Additionally, although they may be of some clinical value, the improvement in overall cancer-related survival and the lack of incorporation of major technological and systemic cancer treatments have limited their reliability and rendered them non-relevant in the current era of metastatic spinal tumor treatment (1).

\section{New treatment options}

Currently, new treatment modalities have improved the management of vertebral metastases. Targeted therapies, biologics and checkpoint inhibitors have markedly improved the overall and progression-free survival for the majority of solid tumors and hematological malignancies (1). Surgical advances, including modern spinal implants and minimally invasive techniques, such as separation surgery, pedicle screw fixation (open or percutaneous), radiofrequency ablation and cement augmentation procedures with polymethylmethacrylate (vertebroplasty and percutaneous balloon kyphoplasty), result in lower surgery-related morbidity and rapid continuation of systemic therapies (1). Furthermore, the evolution and integration of spinal stereotactic radiosurgery, which delivers high-dose conformal radiation in ablative doses, has also notably improved local control rates, regardless of tumor histology, size and radiosensitivity (13).

\section{NOMS algorithm}

The NOMS decision algorithm, which was introduced by the interdisciplinary spine team at Memorial Sloan-Kettering Cancer Center in order to provide a decision-making tool for the management of metastatic spinal tumor, is based on four essential pillars: Neurological and oncological status, mechanical instability and systemic disease assessment $(1,14)$. Based on the evaluation of these four parameters, the interdisciplinary team may design the optimal treatment involving radiotherapy, surgery, systemic therapy or a combination of those. Unlike traditional scoring systems, the NOMS algorithm accommodates the new developments in oncology as they become available, and aims to optimize local tumor control, pain relief and neurological function, while minimizing the risk of morbidity and mortality $(1,13,14)$.

Briefly, in NOMS, the neurological and oncological assessment are jointly considered. The neurological indications are based on the clinical presence of myelopathy or functional radiculopathy and the degree of epidural spinal canal compromise. Epidural spinal cord compression is calculated by a validated six-point scoring system using MR axial T2-weighed images at the site of most critical compression. This scoring system is used to distinguish nil or minimal compression (0-1c) from high-grade epidural spinal compression $(2-3)(1,13,14)$. The oncological assessment evaluates the predicted local tumor response to radiation, chemotherapy, targeted therapy, immunotherapy or hormonal therapy, and it practically reflects the radiosensitivity of the tumors to conventional radiation $(1,14)$. 
In contrast to previously reported scores, in NOMS, framework mechanical instability, as defined by Spinal Instability Neoplastic Score (SINS), is an independent indication for surgical stabilization or percutaneous cement augmentation, regardless of the grade of the epidural spinal cord compression and the radiosensitivity of the tumor (1). Low SINS (0-6) generally suggests a stable lesion, while high-SINS (13-18) tumors are considered unstable, requiring surgical stabilization. In intermediate-SINS tumors (7-12), the need for treatment is based on the discretion and the experience of the spinal surgeon (15). The fourth element of consideration in the NOMS algorithm is the extent of the systemic disease. The medical comorbidities, the ability of the patient to tolerate a recommended procedure, the risk-benefit ratio of treatment and the overall patient survival are considered in the decision-making process $(1,14)$.

\section{Future perspectives}

Modern clinical practice in oncology warrants accurate, reproducible and easily applied prognostication models that can be used both as guidance for decision-making and as scientific tools for research and clinical audits. The development of a variety of scoring systems over the last 30 years has uncovered the need for such a prognostic instrument and the inherent imperfections of the traditional tools (9). Moreover, given the rapid medical and technological advances in the field of cancer treatment, conventional scoring systems appear insufficient for guiding surgical decisions and have become obsolete, underlying the urgent need for modern prognostication tools (1). Within these considerations, future challenges include the development of personalized scoring systems that correspond to the histology of the primary tumor and the specific genetic and anatomical prerequisites of particular tumors and individual patients $(7,9)$. The biology of the tumor is of utmost importance, but in cases of urgent surgical intervention due to neurological deficit, the type of the tumor is not among the primary considerations. Different types of cancer, or cancers with a distinct genetic footprint, would likely intensify the evolution of individualized scoring systems that incorporate specific markers of particular types of malignancies as prognostic factors (7). Finally, an interdisciplinary approach is mandatory in order to design new scoring systems taking into account the recent survival improvement achieved by the advances in cancer management, such as minimally invasive surgical techniques, stereotactic radiotherapy and targeted therapies, among others (7).

There were certain limitations to the present review, as it did not perform a one-stop comparison between the strengths and weaknesses of different scoring systems, and did not provide tables comparing the different parameters of each system; however, it highlights the need for further improvement of scoring systems by taking into account the evolution of cancer diagnostic and therapeutic methods.

\section{Conclusions}

Despite major medical accomplishments in the management of metastatic cancer, surgery, although high-risk, remains crucial for patients with vertebral metastases, particularly those with high-grade epidural spinal cord compression and those requiring spinal stabilization as determined by SINS. Scoring systems for spinal metastases have been proposed as prognostic models to improve the referral pattern and outcomes of surgery and to avoid mistreatment, overtreatment or severe surgical complications. Currently, the NOMS framework appears to offer a reliable, reproducible, patient-tailored multidisciplinary approach to the treatment of spinal metastatic tumors. Prognostication models should continuously evolve by employing more oncological perspectives and incorporating major advances in cancer care.

\section{Acknowledgements}

Not applicable.

\section{Funding}

No funding was received.

\section{Availability of data and materials}

Not applicable.

\section{Authors' contributions}

KA and CK conceived the current study. AI, KR and ES searched the literature. KA, AI, KR and ES drafted the manuscript. CK corrected the manuscript.

\section{Ethics approval and consent to participate}

Not applicable.

\section{Patient consent for publication}

Not applicable.

\section{Competing interests}

The authors declare that they have no competing interests.

\section{References}

1. Barzilai O, Fisher CG and Bilsky MH: State of the Art treatment of spinal metastatic disease. Neurosurgery 82: 757-769, 2018.

2. Delank KS, Wendtner C, Eich HT and Eysel P: The treatment of spinal metastases. Dtsch Arztebl Int 108: 71-80, 2011.

3. Papastefanou S, Alpantaki K, Akra G and Katonis P: Predictive value of Tokuhashi and Tomita scores in patients with metastatic spine disease. Acta Orthop Traumatol Turc 46: 50-56, 2012.

4. Deng Z, Xu B, Jin J, Zhao J and Xu H: Strategies for management of spinal metastases: A comprehensive review. Cancer Transl Med 1: 94-100, 2015.

5. Klimo P Jr, Thompson CJ, Kestle JR and Schmidt MH: A meta-analysis of surgery versus conventional radiotherapy for the treatment of metastatic spinal epidural disease. Neuro Oncol 7: 64-76, 2006.

6. Jansson KA and Bauer HC: Survival, complications and outcome in 282 patients operated for neurological deficit due to thoracic or lumbar spinal metastases. Eur Spine J 15: 196-202, 2006.

7. Tokuhashi Y, Uei H, Oshima M and Ajiro Y: Scoring system for prediction of metastatic spine tumor prognosis. World $\mathrm{J}$ Orthop 5: 262-271, 2014. 
8. Ragel BT, Mendez GA, Reddington J, Ferachi D, Kubicky CD, Philipp TC, Zusman NL, Klimo P, Hart R, Yoo J and Ching AC: Life expectancy and metastatic spine scoring systems: An academic institutional experience. Clin Spine Surg 30: 335-342, 2017.

9. Cassidy JT, Baker JF and Lenehan B: The role of prognostic scoring systems in assessing surgical candidacy for patients with vertebral metastasis: A narrative review. Global Spine J 8: 638-651, 2018.

10. Engelhardt EG, Révész D, Tamminga HJ, Punt CJA, Koopman M, Onwuteaka-Philipsen BD, Steyerberg EW, Jansma IP, De Vet HCW and Coupé VMH: Clinical usefulness of tools to support decision-making for palliative treatment of metastatic colorectal cancer: A systematic review clinical colorectal cancer. Clin Colorectal Cancer 17: e1-e12, 2018.

11. Tokuhashi Y, Matsuzaki H, Toriyama S, Kawano H and Ohsaka S: Scoring system for the preoperative evaluation of metastatic spine tumor prognosis. Spine (Phila Pa 1976) 15: 1110-1113, 1990.
12. Tomita K, Kawahara N, Kobayashi T, Yoshida A, Murakami H and Akamaru T: Surgical strategy of spinal metastases. Spine (Phila Pa 1976) 26: 298-306, 2001.

13. Joaquim AF, Powers A, Laufer I and Bilsky MH: An update in the management of spinal metastases. Arq Neuropsiquiatr 73: 795-802, 2015.

14. Laufer I, Rubin DG, Lis E, Cox BW, Stubblefield MD, Yamada Y and Bilsky MH: The NOMS framework: Approach to the treatment of spinal metastatic tumors. Oncologist 18: 744-751, 2013.

15. Fourney DR, Frangou EM, Ryken TC, Dipaola CP, Shaffrey CI, Berven SH, Bilsky MH, Harrop JS, Fehlings MG, Boriani S, et al: Spinal instability neoplastic score: An analysis of reliability and validity from the spine oncology study group. J Clin Oncol 29: 3072-3077, 2011 